PROCEEDINGS OF THE

AMERICAN MATHEMATICAL SOCIETY

Volume 134, Number 1, Pages 243-251

S 0002-9939(05)07954-2

Article electronically published on June 13, 2005

\title{
CONVERGENCE THEOREM FOR ZEROS OF GENERALIZED LIPSCHITZ GENERALIZED PHI-QUASI-ACCRETIVE OPERATORS
}

\author{
C. E. CHIDUME AND C. O. CHIDUME
}

(Communicated by Joseph A. Ball)

\begin{abstract}
Let $E$ be a uniformly smooth real Banach space and let $A: E \rightarrow E$ be a mapping with $N(A) \neq \emptyset$. Suppose $A$ is a generalized Lipschitz generalized $\Phi$-quasi-accretive mapping. Let $\left\{a_{n}\right\},\left\{b_{n}\right\}$, and $\left\{c_{n}\right\}$ be real sequences in $[0,1]$ satisfying the following conditions: (i) $a_{n}+b_{n}+c_{n}=1$; (ii) $\sum\left(b_{n}+c_{n}\right)=$ $\infty$; (iii) $\sum c_{n}<\infty$; (iv) $\lim b_{n}=0$. Let $\left\{x_{n}\right\}$ be generated iteratively from arbitrary $x_{0} \in E$ by

$$
x_{n+1}=a_{n} x_{n}+b_{n} S x_{n}+c_{n} u_{n}, n \geq 0,
$$

where $S: E \rightarrow E$ is defined by $S x:=x-A x \forall x \in E$ and $\left\{u_{n}\right\}$ is an arbitrary bounded sequence in $E$. Then, there exists $\gamma_{0} \in \Re$ such that if $b_{n}+c_{n} \leq \gamma_{0} \forall n \geq 0$, the sequence $\left\{x_{n}\right\}$ converges strongly to the unique solution of the equation $A u=0$. A related result deals with approximation of the unique fixed point of a generalized Lipschitz and generalized $\phi$-hemicontractive mapping.
\end{abstract}

\section{INTRODUCTION}

Let $E$ be a real Banach space and let $E^{*}$ be its dual space. The normalized duality mapping $J: E \rightarrow 2^{E^{*}}$ is defined by $J x=\left\{u \in E^{*}:\langle x, u\rangle=\|x\|\|u\|,\|u\|=\right.$ $\|x\|\}$, where $\langle$,$\rangle denotes the generalized duality pairing.$

Definition 1.1. A mapping $A$ with domain $D(A)$ and range $R(A)$ in $E$ is said to be strongly accretive if there exists a constant $k \in(0,1)$ such that for all $x, y \in D(A)$, there exists $j(x-y) \in J(x-y)$ such that $\langle A x-A y, j(x-y)\rangle \geq k\|x-y\|^{2}$, and is called $\phi$-strongly accretive (see, e.g., Deimling [6]) if there is a strictly increasing function $\phi:[0, \infty) \rightarrow[0, \infty)$ with $\phi(0)=0$ such that for any $x, y \in D(A)$ there exists $j(x-y) \in J(x-y)$ such that

$$
\langle A x-A y, j(x-y)\rangle \geq \phi(\|x-y\|)\|x-y\| .
$$

The mapping $A$ is called generalized $\Phi$-accretive if there exists a strictly increasing function $\phi:[0, \infty) \rightarrow[0, \infty)$ with $\phi(0)=0$ such that for all $x, y \in D(A)$ there exists $j(x-y) \in J(x-y)$ such that

$$
\langle A x-A y, j(x-y)\rangle \geq \Phi(\|x-y\|) .
$$

Received by the editors August 2, 2004 and, in revised form, August 30, 2004.

2000 Mathematics Subject Classification. Primary 47H09, 47J25.

Key words and phrases. Generalized Lipschitz maps, generalized $\Phi$-quasi-accretive maps, generalized $\Phi$-hemicontractive maps, uniformly smooth real Banach spaces. 
It is well known that the class of generalized $\Phi$-accretive mappings includes the class of $\phi$-strongly accretive operators as a special case (one sets $\Phi(s)=s \phi(s)$ for all $s \in[0, \infty))$.

Let $N(A):=\{x \in D(A): A x=0\} \neq \emptyset$.

Definition 1.2. The mapping $A$ is called strongly quasi-accretive if there exists $k \in(0,1)$ such that for all $x \in D(A), x^{*} \in N(A)$ there exists $j\left(x-x^{*}\right) \in J\left(x-x^{*}\right)$ such that $\left\langle A x-A x^{*}, j\left(x-x^{*}\right)\right\rangle \geq k\left\|x-x^{*}\right\|^{2}$. $A$ is called $\phi$-strongly quasi-accretive if there exists a strictly increasing function $\phi:[0, \infty) \rightarrow[0, \infty)$ with $\phi(0)=0$ such that for all $x \in D(A), x^{*} \in N(A)$ there exists $j\left(x-x^{*}\right) \in J\left(x-x^{*}\right)$ such that $\left\langle A x-A x^{*}, j\left(x-x^{*}\right)\right\rangle \geq \phi\left(\left\|x-x^{*}\right\|\right)\left\|x-x^{*}\right\|$. Finally, $A$ is called generalized $\Phi-$ quasi-accretive if there exists a strictly increasing function $\Phi:[0, \infty) \rightarrow[0, \infty)$ with $\Phi(0)=0$ such that for all $x \in D(A), x^{*} \in N(A)$ there exists $j\left(x-x^{*}\right) \in J\left(x-x^{*}\right)$ such that $\left\langle A x-A x^{*}, j\left(x-x^{*}\right)\right\rangle \geq \Phi\left(\left\|x-x^{*}\right\|\right)$.

Definition 1.3. A mapping $G: E \rightarrow E$ is called Lipschitz if there exists a constant $L>0$ such that $\|G x-G y\| \leq L\|x-y\| \forall x, y \in D(G)$.

Closely related to the class of accretive-type mappings are those of pseudocontractive types.

Definition 1.4. A mapping $T: E \rightarrow E$ is called strongly pseudo-contractive if and only if $(I-T)$ is strongly accretive, and is called strongly $\phi$-pseudo-contractive if and only if $(I-T)$ is $\phi$-strongly accretive. The mapping $T$ is called generalized $\Phi$-pseudo-contractive if and only if $(I-T)$ is generalized $\Phi$-accretive.

Definition 1.5. If $F(T):=\{x \in E: T x=x\} \neq \emptyset$, the mapping $T$ is called strongly hemi-contractive if and only if $(I-T)$ is strongly quasi-accretive; it is called $\phi$-hemi-contractive if and only if $(I-T)$ is $\phi$-strongly quasi-accretive; and $T$ is called generalized $\Phi$-hemi-contractive if and only if $(I-T)$ is generalized $\Phi$ quasi-accretive.

The class of generalized $\Phi$-hemi-contractive mappings is the most general (among those defined above) for which $T$ has a unique fixed point. For a comparison of the relationships between the various classes of nonlinear mappings defined above, the reader may consult [2].

The intimate connection between the zeros of accretive-type operators and the fixed points of pseudo-contractive-type mappings is well known. The accretive-type operators are deeply connected with nonlinear equations of evolution (see, e.g., [1], [6] ).

It is well known that in many evolution systems, zeros of accretive-type operators (or, equivalently, fixed points of pseudo-contractive-type mappings) correspond to equilibrium points of the systems. Consequently, methods of approximating these zeros or fixed points have been a flourishing area for many researchers in nonlinear operator theory.

Numerous convergence results have been proved on iterative methods for approximating zeros of Lipschitz accretive-type (or pseudo-contractive type) nonlinear mappings (see, e.g., [2], 3], 4], [5], [7, 8], 14, and the references contained therein). Also, many authors have proved convergence theorems under the assumption that these operators have bounded range (see, e.g., [7]). A natural generalization of the class of Lipschitz mappings and the class of mappings with bounded 
range is that of a generalized Lipschitz map. A mapping $S: D(S) \subset E \rightarrow E$ is called a generalized Lipschitz map if there exists a constant $L>0$ such that

$$
\|S x-S y\| \leq L(1+\|x-y\|) \forall x, y \in D(S) .
$$

Clearly, every Lipschitz map is a generalized Lipschitz map. Furthermore, every map with a bounded range is a generalized Lipschitz map. The following example (see, e.g., [2]) shows that the class of generalized Lipschitz maps properly includes the class of Lipschitz maps and that of mappings with bounded range.

Example. Let $E=(-\infty, \infty)$ and let $T: E \rightarrow E$ be defined by

$$
T x= \begin{cases}x-1 & \text { if } x \in(-\infty,-1), \\ x-\sqrt{1-(x+1)^{2}} & \text { if } x \in[-1,0), \\ x+\sqrt{1-(x-1)^{2}} & \text { if } x \in[0,1], \\ x+1 & \text { if } x \in(1, \infty) .\end{cases}
$$

Clearly, $T$ is a generalized Lipschitz map which is not Lipschitz and whose range is not bounded. The following interesting results have recently been proved.

Theorem HH1 (8, Corollary 1]). Let $E$ be a uniformly smooth Banach space and let $T: E \rightarrow 2^{E}$ be a multivalued $\phi$-hemi-contractive operator with bounded range. Suppose $\left\{a_{n}\right\},\left\{b_{n}\right\},\left\{c_{n}\right\}$ are real sequences in $[0,1]$ satisfying the following conditions: (i) $a_{n}+b_{n}+c_{n}=1 \forall n \geq 0$; (ii) $\lim b_{n}=0$; (iii) $\sum b_{n}=\infty$; (iv) $c_{n}=o\left(b_{n}\right)$. For arbitrary $x_{1}, u_{1} \in E$, define the sequence $\left\{x_{n}\right\}$ by

$$
x_{n+1}=a_{n} x_{n}+b_{n} \nu_{n}+c_{n} u_{n}, \exists \nu_{n} \in T x_{n}, n \geq 0,
$$

where $\left\{u_{n}\right\}$ is an arbitrary bounded sequences in E. Then, $\left\{x_{n}\right\}$ converges strongly to the unique fixed point of $T$.

Theorem HH2 ([8]). Let $E$ be a uniformly smooth Banach space and let $T$ : $E \rightarrow 2^{E}$ be a multivalued $\phi$-strongly accretive operator with bounded range. For a given $f \in E$, let $x^{*}$ denote a solution of the inclusion $f \in T x$. Define the operator $S: E \rightarrow E$ by sx $=f+x-T x$. Suppose $\left\{a_{n}\right\},\left\{b_{n}\right\},\left\{c_{n}\right\}$ are real sequences in $[0,1]$ satisfying conditions (i)-(iv) of Theorem HH1. For arbitrary $x_{1}, u_{1} \in E$, define the sequence $\left\{x_{n}\right\}$ by

$$
x_{n+1}=a_{n} x_{n}+b_{n} \nu_{n}+c_{n} u_{n}, \exists \nu_{n} \in S x_{n}, n \geq 0,
$$

where $\left\{u_{n}\right\}$ is an arbitrary bounded sequence in $E$. Then, $\left\{x_{n}\right\}$ converges strongly to the unique solution of the operator equation $f \in T x$.

Theorem HH2 is an immediate corollary of Theorem 2 of [8. Theorems HH1 and HH2 include several recent important results as special cases (see, e.g., Remarks 1, 2 and 3 in 8 ).

It is our purpose in this paper to prove convergence theorems for zeros of generalized Lipschitz, generalized $\Phi$-quasi-accretive operators and for fixed points of generalized Lipschitz, generalized $\Phi$-hemi-contractive mappings. The Mann iteration process introduced by $\mathrm{Xu} 13$ is the one studied in theorems HH1, HH2 and in the theorems of this paper. Our theorems provide significant improvements of theorems $\mathrm{HH} 1$ and $\mathrm{HH} 2$. 


\section{Preliminaries}

Let $E$ be a normed space with $\operatorname{dim} E \geq 2$. The modulus of smoothness of $E$ is the function $\rho_{E}:[0, \infty) \rightarrow[0, \infty)$ defined by

$$
\rho_{E}(\tau):=\sup \left\{\frac{\|x+y \mid+\| x-y \|}{2}-1:\|x\|=1 ;\|y\|=\tau\right\} .
$$

Clearly, $\rho_{E}(0)=0$. The space $E$ is called uniformly smooth if and only if

$$
\lim _{t \rightarrow 0^{+}} \frac{\rho_{E}(t)}{t}=0
$$

In the sequel, we shall make use of the following well-known result.

Lemma. Let $E$ be a real normed linear space. Then, the following inequality holds:

$$
\|x+y\|^{2} \leq\|x\|^{2}+2\langle y, j(x+y)\rangle \forall x, y \in E, \forall j(x+y) \in J(x+y) .
$$

\section{Main Results}

In the sequel, we shall assume, without loss of generality, that $f=0$.

Theorem 3.1. Let $E$ be a uniformly smooth real Banach space and let $A: E \rightarrow E$ be a mapping with $N(A) \neq \emptyset$. Suppose $A$ is a generalized Lipschitz generalized $\Phi$-quasi-accretive mapping. Let $\left\{a_{n}\right\},\left\{b_{n}\right\}$, and $\left\{c_{n}\right\}$ be real sequences in $[0,1]$ satisfying the following conditions: (i) $a_{n}+b_{n}+c_{n}=1$; (ii) $\sum\left(b_{n}+c_{n}\right)=\infty$; (iii) $\sum_{b y} c_{n}<\infty$; (iv) $\lim b_{n}=0$. Let $\left\{x_{n}\right\}$ be generated iteratively from arbitrary $x_{0} \in E$

$$
x_{n+1}=a_{n} x_{n}+b_{n} S x_{n}+c_{n} u_{n}, n \geq 0,
$$

where $S: E \rightarrow E$ is defined by $S x:=x-A x \forall x \in E$ and $\left\{u_{n}\right\}$ is an arbitrary bounded sequence in $E$. Then, there exists $\gamma_{0} \in \Re$ such that if $b_{n}+c_{n} \leq \gamma_{0} \forall n \geq 0$, the sequence $\left\{x_{n}\right\}$ converges strongly to the unique solution of the equation $A u=0$.

Proof. Let $x^{*} \in N(A)$ be arbitrary. Observe that $A x^{*}=0$ if and only if $S x^{*}=x^{*}$. Since $A$ is a generalized Lipschitz, generalized $\Phi$-quasi-accretive mapping, there exist a strictly increasing function $\Phi:[0, \infty) \rightarrow[0, \infty), \Phi(0)=0$, and $j\left(x-x^{*}\right) \in$ $J\left(x-x^{*}\right)$ such that the following inequalities hold:

$$
\left\|S x_{n}-S x^{*}\right\| \leq L\left(1+\left\|x_{n}-x^{*}\right\|\right)
$$

and

$$
\left\langle S x_{n}-S x^{*}, j\left(x_{n}-x^{*}\right)\right\rangle \leq\left\|x_{n}-x^{*}\right\|^{2}-\Phi\left(\left\|x_{n}-x^{*}\right\|\right) .
$$

Uniqueness of the fixed point follows from inequality (4), as in [8]. Set $\alpha_{n}:=b_{n}+c_{n}$. Then, the recursion formula (2) reduces to the formula

$$
x_{n+1}=\left(1-\alpha_{n}\right) x_{n}+\alpha_{n} S x_{n}+U_{n}, \neq 0,
$$

where $U_{n}:=c_{n}\left(u_{n}-S x_{n}\right)$.

We first prove that the sequence $\left\{x_{n}\right\}$ is bounded.

If $x_{0}=S x_{n} \forall n \geq 0$, then we are done. Suppose this is not the case, i.e., suppose there exists a smallest positive natural number $n_{0}$ such that $x_{n_{0}} \neq S x_{n_{0}}$. Without loss of generality, set $x_{n_{0}}=x_{0}$. For $x_{0} \in E$, define $a_{0}:=\left\|x_{0}-S x_{0}\right\| \cdot\left\|x_{0}-x^{*}\right\|$. From

$$
\left\langle S x_{0}-S x^{*}, j\left(x_{0}-x^{*}\right)\right\rangle \leq\left\|x_{0}-x^{*}\right\|^{2}-\Phi\left(\left\|x_{0}-x^{*}\right\|\right),
$$


we obtain that

$$
\left\|x_{0}-x^{*}\right\| \leq \Phi^{-1}\left(a_{0}\right) .
$$

Since $j$ is uniformly continuous on bounded subsets of $E$, given

$$
\epsilon_{0}:=\frac{a_{0}}{2\left[2(1+L) \Phi^{-1}\left(a_{0}\right)+L\right]}>0, \exists \delta>0,
$$

such that $\|x-y\|<\delta \Rightarrow\|j(x)-j(y)\|<\epsilon \forall x, y \in B_{R}(0)$, for some $R>0$. Set $N^{*}:=\sup _{n}\left\|u_{n}-x^{*}\right\|$ and define

$$
\gamma_{0}:=\min \frac{1}{2}\left\{1, \frac{\Phi^{-1}\left(a_{0}\right)}{3\left[2(1+2 L) \Phi^{-1}\left(a_{0}\right)+2 L\right]}, \frac{\delta}{2(1+L) \Phi^{-1}\left(a_{0}\right)+L}, \frac{\Phi^{-1}\left(a_{0}\right)}{3 N^{*}}\right\} .
$$

Claim 1. $\left\|x_{n}-x^{*}\right\| \leq 2 \Phi^{-1}\left(a_{0}\right) \forall n \geq 0$.

The claim clearly holds for $n=0$. Assume $\left\|x_{n}-x^{*}\right\| \leq 2 \Phi^{-1}\left(a_{0}\right)$ holds for some $n$. We prove $\left\|x_{n+1}-x^{*}\right\| \leq 2 \Phi^{-1}\left(a_{0}\right)$. Suppose this is not the case, i.e., suppose that $\left\|x_{n+1}-x^{*}\right\|>2 \Phi^{-1}\left(a_{0}\right)$. Then, we obtain the following inequalities:

(a) $\left\|x_{n}-S x_{n}\right\| \leq\left[2(1+L) \Phi^{-1}\left(a_{0}\right)+L\right]$.

(b) $\left\|x_{n+1}-x^{*}\right\| \leq 3 \Phi^{-1}\left(a_{0}\right)$.

(c) $\left\|x_{n}-x^{*}\right\| \geq \Phi^{-1}\left(a_{0}\right)$.

We also obtain the following inequality:

(d) $\left\|U_{n}\right\| .\left\|x_{n}-x^{*}\right\| \leq c_{n} M_{1}$, where $M_{1}:=\left[N^{*}+L\left(1+2 \Phi^{-1}\left(a_{0}\right)\right)\right] 2 \Phi^{-1}\left(a_{0}\right)$.

Using the recursion formula (5), we compute as follows:

$$
\begin{aligned}
\left\|x_{n+1}-x^{*}\right\|^{2}= & \left\|x_{n}-x^{*}-\left[\alpha_{n}\left(x_{n}-S x_{n}\right)-U_{n}\right]\right\|^{2} \\
\leq & \left\|x_{n}-x^{*}\right\|^{2}-2 \alpha_{n}\left\langle x_{n}-S x_{n}, j\left(x_{n+1}-x^{*}\right)-j\left(x_{n}-x^{*}\right)\right\rangle \\
& +2\left\|U_{n}\right\| \cdot\left\|j\left(x_{n+1}-x^{*}\right)-j\left(x_{n}-x^{*}\right)\right\| \\
& -2 \alpha_{n}\left\langle x_{n}-S x_{n}, j\left(x_{n}-x^{*}\right)\right\rangle+2\left\|U_{n}\right\| \cdot\left\|x_{n}-x^{*}\right\| \\
\leq \quad & \left\|x_{n}-x^{*}\right\|^{2}-2 \alpha_{n} \Phi\left(\left\|x_{n}-x^{*}\right\|\right)+2 \alpha_{n}\left\|x_{n}-S x_{n}\right\| \\
& \times\left\|j\left(x_{n+1}-x^{*}\right)-j\left(x_{n}-x^{*}\right)\right\| \\
& +2\left\|U_{n}\right\| \cdot\left\|j\left(x_{n+1}-x^{*}\right)-j\left(x_{n}-x^{*}\right)\right\|+2\left\|U_{n}\right\| \cdot\left\|x_{n}-x^{*}\right\| \\
\leq \quad & \left\|x_{n}-x^{*}\right\|^{2}-2 \alpha_{n} a_{0}+2 \alpha_{n}\left[2(1+L) \Phi^{-1}\left(a_{0}\right)+L\right] \\
& \times\left\|j\left(x_{n+1}-x^{*}\right)-j\left(x_{n}-x^{*}\right)\right\| \\
& +M_{1} c_{n}+c_{n} 2\left[N^{*}+L\left\{1+2 \Phi^{-1}\left(a_{0}\right)\right\}\right] \\
& .\left\|j\left(x_{n+1}-x^{*}\right)-j\left(x_{n}-x^{*}\right)\right\| .
\end{aligned}
$$

Set $M:=M_{1}+2\left[N^{*}+L\left\{1+2 \Phi^{-1}\left(a_{0}\right)\right\}\right] \epsilon_{0}$. Observe that $\left(x_{n+1}-x^{*}\right),\left(x_{n}-x^{*}\right) \in$ $B_{R}(0)$, where $R:=3 \Phi^{-1}\left(a_{0}\right)>0$. Moreover, $\left\|x_{n+1}-x_{n}\right\|=\alpha_{n}\left\|x_{n}-S x_{n}\right\| \leq$ $\gamma_{0}\left[2(1+L) \Phi^{-1}\left(a_{0}\right)+L\right]<\delta$, so that $\left\|j\left(x_{n+1}-x^{*}\right)-j\left(x_{n}-x^{*}\right)\right\|<\epsilon_{0}$. Hence, we obtain that

$$
\left\|x_{n+1}-x^{*}\right\|^{2} \leq\left\|x_{n}-x^{*}\right\|^{2}-\alpha_{n} a_{0}+c_{n} M,
$$

and this implies that

$$
a_{0} \sum_{j=0}^{n} \alpha_{j} \leq \sum_{j=0}^{n}\left(\left\|x_{j}-x^{*}\right\|^{2}-\left\|x_{j+1}-x^{*}\right\|^{2}\right)+M \sum_{j=0}^{n} c_{j}<\infty
$$

(since $\sum_{n=0}^{\infty} c_{n}<\infty$ ), contradicting condition (ii) of the theorem. Hence, claim 1 holds and so, $\left\{x_{n}\right\}$ is bounded. Since $S$ is a bounded operator, $\left\{S x_{n}\right\}$ is also 
bounded. Set

$$
\begin{gathered}
\rho:=\sup \left\|x_{n}-S x_{n}\right\| ; \beta:=\sup \left\|u_{n}-S x_{n}\right\|, \\
\sigma:=\sup \left\{2\left\|u_{n}-S x_{n}\right\| .\left\|j\left(x_{n+1}-x^{*}\right)-j\left(x_{n}-x^{*}\right)\right\|+2\left\|u_{n}-S x_{n}\right\| .\left\|x_{n}-x^{*}\right\|\right\} .
\end{gathered}
$$

Then,

$$
\begin{aligned}
\left\|x_{n+1}-x^{*}\right\|^{2} \leq & \left\|x_{n}-x^{*}\right\|^{2}-2 \alpha_{n} \Phi\left(\left\|x_{n}-x^{*}\right\|\right)+2 \alpha_{n}\left\|x_{n}-S x_{n}\right\| \\
& \times\left\|j\left(x_{n+1}-x^{*}\right)-j\left(x_{n}-x^{*}\right)\right\| \\
& +2\left\|U_{n}\right\| \cdot\left\|j\left(x_{n+1}-x^{*}\right)-j\left(x_{n}-x^{*}\right)\right\|+2\left\|U_{n}\right\| .\left\|x_{n}-x^{*}\right\| \\
\leq & \left\|x_{n}-x^{*}\right\|^{2}-2 \alpha_{n} \Phi\left(\left\|x_{n}-x^{*}\right\|\right) \\
& +2 \alpha_{n} \rho .\left\|j\left(x_{n+1}-x^{*}\right)-j\left(x_{n}-x^{*}\right)\right\|+c_{n} \sigma .
\end{aligned}
$$

Claim 2. $\inf \left\{\left\|x_{n}-x^{*}\right\|, n \geq 0\right\}=0$.

Suppose this is not the case. Let inf $\left\{\left\|x_{n}-x^{*}\right\|, n \geq 0\right\}=\delta>0$. Then, $\| x_{n}-x^{*}||>\delta \forall n \geq 0$. This implies that $\Phi\left(\| x_{n}-x^{*}||\right)>\Phi(\delta) \forall n \geq 0$. Furthermore, since $\left\|x_{n+1}-x_{n}\right\|=\alpha_{n}\left\|x_{n}-S x_{n}\right\|+c_{n}\left\|u_{n}-S x_{n}\right\|=\rho \alpha_{n}+\beta c_{n} \rightarrow 0$ as $n \rightarrow \infty$, and $j$ is uniformly continuous on bounded subsets of $E$, there exists an integer $N_{1}>N_{0}$ such that $\left\|j\left(x_{n+1}-x^{*}\right)-j\left(x_{n}-x^{*}\right)\right\|<\frac{\Phi(\delta)}{2 \rho} \forall n \geq N_{1}$. Hence, for all $n \geq N_{1}$, we obtain that

$$
\left\|x_{n+1}-x^{*}\right\|^{2} \leq\left\|x_{n}-x^{*}\right\|^{2}-\Phi(\delta) \alpha_{n}+\sigma c_{n}, \forall n \geq N_{1} .
$$

This implies that

$$
\Phi(\delta) \sum_{j=0}^{n} \alpha_{j} \leq \sum_{j=0}^{n}\left(\left\|x_{j}-x^{*}\right\|^{2}-\left\|x_{j+1}-x^{*}\right\|^{2}\right)+\sigma \sum_{j=0}^{n} c_{j},
$$

which, since $\sum_{n=0}^{\infty} c_{n}<\infty$, implies that $\sum_{n=0}^{\infty} \alpha_{n}<\infty$, contradicting condition (ii). Hence, Claim 2 holds. Consequently, there exists a subsequence $\left\{x_{n_{i}}\right\}$ of $\left\{x_{n}\right\}$ such that $\lim _{i \rightarrow \infty}\left\|x_{n_{i}}-x^{*}\right\|=0$. Let $\epsilon>0$ be given. Choose $N_{2}>N_{1}$ such that, for all $n \geq N_{2}$,

$$
\rho \alpha_{n}<\frac{\epsilon}{4}, \beta c_{n}<\frac{\epsilon}{4}, c_{n}=\min \left\{\frac{\epsilon}{4 \beta}, \frac{1}{2 \sigma} \Phi\left(\frac{\epsilon}{2}\right) \alpha_{n}\right\} .
$$

Choose an integer $N_{3}>N_{2}$ such that

$$
\left\|j\left(x_{n_{i}+1}-x^{*}\right)-j\left(x_{n_{i}}-x^{*}\right)\right\| \leq \frac{\Phi\left(\frac{\epsilon}{2}\right)}{2 \rho} .
$$

Now, choose an integer $N_{4} \geq N_{3}$ such that $\left\|x_{n_{i}}-x^{*}\right\|<\epsilon \forall i \geq N_{4}$. Fix $i_{*} \geq N_{4}$. In particular,

$$
\left\|x_{n_{i_{*}}}-x^{*}\right\|<\epsilon
$$

\section{Claim 3.}

$$
\| x_{n_{i *}+m}-x^{*}||<\epsilon \forall m=1,2, \ldots .
$$

The proof of this claim is by induction. We first prove that $\left\|x_{n_{i *}+1}-x^{*}\right\|<\epsilon$. Suppose this is not the case. Then, $\left\|x_{n_{i *}+1}-x^{*}\right\| \geq \epsilon$. Using the recursion formula (5) we obtain that

$$
\left\|x_{n_{i *}}-x^{*}\right\| \geq \epsilon-\rho \alpha_{n_{i}}-\beta c_{n_{i}}>\frac{\epsilon}{2} .
$$


This implies $\Phi\left(\left\|x_{n_{i *}}-x^{*}\right\|\right) \geq \Phi\left(\frac{\epsilon}{2}\right), \forall n \geq N_{4}$. Using the recursion formula (5) again, we now obtain the following estimates:

$$
\left\|x_{n_{i *}+1}-x^{*}\right\|^{2} \leq\left\|x_{n_{i *}}-x^{*}\right\|^{2}-\frac{1}{2} \alpha_{n_{i *}} \Phi\left(\frac{\epsilon}{2}\right)<\epsilon^{2},
$$

a contradiction. Hence Claim 3 holds for $m=1$. Assume now it holds for $m=k$. Following the above argument, one easily proves that it holds for $m=k+1$. Hence, Claim 2 holds. This shows that $\left\{x_{n}\right\}$ converges strongly to $x^{*}$ as $n \rightarrow \infty$, completing proof of Theorem 3.1.

Theorem 3.2. Let $E$ be a uniformly smooth real Banach space. Let $F(T):=\{x \in$ $E: T x=x\} \neq \emptyset$. Suppose $T: E \rightarrow E$ is a generalized Lipschitz and generalized $\Phi$-hemi-contractive mapping. Let $\left\{a_{n}\right\},\left\{b_{n}\right\}$, and $\left\{c_{n}\right\}$ be real sequences in $[0,1)$ satisfying the following conditions: (i) $a_{n}+b_{n}+c_{n}=1$; (ii) $\sum\left(b_{n}+c_{n}\right)=\infty$; (iii) $\sum_{b y} c_{n}<\infty$; (iv) $\lim b_{n}=0$. Let $\left\{x_{n}\right\}$ be generated iteratively from arbitrary $x_{0} \in E$

$$
x_{n+1}=a_{n} x_{n}+b_{n} T x_{n}+c_{n} u_{n}, n \geq 0,
$$

where $\left\{u_{n}\right\}$ is an arbitrary bounded sequence in $E$. Then, there exists $\gamma_{0} \in \Re$ such that if $b_{n}+c_{n} \leq \gamma_{0} \forall n \geq 0$, the sequence $\left\{x_{n}\right\}$ converges strongly to the unique fixed point of $T$.

Proof. Since $T$ is a generalized $\Phi$-hemi-contractive mapping, it follows that

$$
\left\langle T x-T x^{*}, j\left(x-x^{*}\right)\right\rangle \leq\left\|x-x^{*}\right\|^{2}-\Phi\left(\left\|x-x^{*}\right\|\right) \forall x \in E, x^{*} \in F(T) .
$$

In particular,

$$
\left\langle T x_{n}-T x^{*}, j\left(x_{n}-x^{*}\right)\right\rangle \leq\left\|x_{n}-x^{*}\right\|^{2}-\Phi\left(\left\|x_{n}-x^{*}\right\|\right) .
$$

Since $T$ is generalized Lipschitz, we have

$$
\left\|T x_{n}-T x^{*}\right\| \leq L\left(1+\left\|x_{n}-x^{*}\right\|\right) .
$$

The rest now follows exactly as in the proof of Theorem 3.1, with $S$ replaced by $T$.

Remark 3.3. In 1974 when the Ishikawa iteration process [9] was introduced to successfully approximate fixed points of Lipschitz pseudo-contractive mappings defined on compact convex subsets of Hilbert spaces, it was not known whether or not the simpler Mann process [1] would converge for this class of mappings. For several classes of operators less general than the class of Lipschitz pseudo-contractive mappings, it was discovered that both the Mann and the Ishikawa iteration processes converged. In such cases, it was then of interest to compare the rate of convergence of the two schemes (see, e.g., Rhoades, [12). By the late 1980's and 1990 's, it became clear that whenever both schemes converged, there was no significant difference in their rates of convergence. From then on, it became obvious that whenever the Mann sequence converges, any discussion of the more cumbersome Ishikawa scheme is totally unnecessary. Consequently, we have not discuss the Ishikawa process here.

Remark 3.4. In 1995, Liu 10 introduced what he called the Ishikawa and Mann iteration processes with errors, where the error terms $u_{n}$ and $v_{n}$ satisfy the following conditions: $\sum\left\|u_{n}\right\|<\infty: \sum\left\|v_{n}\right\|<\infty$. There were serious objections to studying these iteration processes because these conditions on the error terms imply, in particular, that the error terms go to zero. This is incompatible with the 
randomness of the occurence of errors. In 1998, Xu 13 introduced a somewhat more satisfactory Ishikawa and Mann processes with error terms $u_{n}$ and $v_{n}$ where these terms are required to be just bounded.

Remark 3.5. Recently, some authors have studied what they call Ishikawa and Mann iterative sequences with mixed errors $u_{n}$ and $v_{n}$, where $u_{n}=u_{n}^{\prime}+u_{n}^{\prime \prime} ; \sum\left\|u_{n}^{\prime}\right\|<$ $\infty ;\left\|u_{n}^{\prime \prime}\right\|=o\left(\alpha_{n}\right)$ and $\left\|v_{n}\right\| \rightarrow 0(n \rightarrow \infty)$. There are many objections to these types of errors.

(i) There is no method of checking that $u_{n}$ is of the form $u_{n}^{\prime}+u_{n}^{\prime \prime}$.

(ii) The conditions $\sum\left\|u_{n}^{\prime}\right\|<\infty$ and $\left\|v_{n}\right\| \rightarrow 0(n \rightarrow \infty)$ have already been objected to in the definition of Liu [10].

(iii) The condition $\left\|u_{n}^{\prime \prime}\right\|=o\left(\alpha_{n}\right)$ not only requires that $u_{n}^{\prime \prime}$ goes to zero as $n$ goes to infinity, but also that it does so faster than $\alpha_{n}$ !!. This is almost impossible to verify in any application.

Remark 3.6. Recently, Xue et al. 15] considered this so-called Ishikawa iterative sequence with mixed errors and proved, in uniformly smooth Banach spaces $E$, that the sequence converges to the unique solution of $x+A x=f$, where $A: E \rightarrow E$ is a generalized Lipschitz $m$-accretive operator. We remark that if one sets $U:=I+A$, then the equation $x+A x=f$ reduces to $U x=f$, where $U$ is strongly accretive. Thus, Xue et al. [15] actually studied the class of strongly accretive mappings. This class of mappings is a very special subclass of the class of generalized $\Phi$-accretive mappings in which $\phi(t):=k t^{2} \forall t \in[0, \infty)$.

Using the technique of this paper and obvious appropriate definitions, the following theorems are easily proved.

Theorem 3.7. Let $E$ be a uniformly smooth real Banach space and let $A: E \rightarrow 2^{E}$ be a multivalued mapping with $N(A) \neq \emptyset$. Suppose $A$ is a generalized Lipschitz generalized $\Phi$-quasi-accretive mapping. Let $\left\{a_{n}\right\},\left\{b_{n}\right\}$, and $\left\{c_{n}\right\}$ be real sequences in $[0,1]$ satisfying the following conditions: (i) $a_{n}+b_{n}+c_{n}=1$; (ii) $\sum\left(b_{n}+c_{n}\right)=\infty$; (iii) $\sum c_{n}<\infty$; (iv) $\lim b_{n}=0$. Let $\left\{x_{n}\right\}$ be generated iteratively from arbitrary $x_{0} \in E$ by

$$
x_{n+1}=a_{n} x_{n}+b_{n} \eta_{n}+c_{n} u_{n}, \exists \eta_{n} \in S x_{n}, \quad n \geq 0,
$$

where $S: E \rightarrow E$ is defined by $S x:=f+x-A x \forall x \in E$ and $\left\{u_{n}\right\}$ is an arbitrary bounded sequence in $E$. Then, there exists $\gamma_{0} \in \Re$ such that if $b_{n}+c_{n} \leq \gamma_{0} \forall n \geq 0$, the sequence $\left\{x_{n}\right\}$ converges strongly to the unique solution of the equation $0 \in A u$.

Theorem 3.8. Let $E$ be a uniformly smooth real Banach space. Let $F(T):=\{x \in$ $E: T x=x\} \neq \emptyset$. Suppose $T: E \rightarrow 2^{E}$ is a multi-valued generalized Lipschitz and generalized $\Phi$-hemi-contractive mapping. Let $\left\{a_{n}\right\},\left\{b_{n}\right\}$, and $\left\{c_{n}\right\}$ be real sequences in $[0,1)$ satisfying the following conditions: (i) $a_{n}+b_{n}+c_{n}=1$; (ii) $\sum\left(b_{n}+c_{n}\right)=\infty$; (iii) $\sum c_{n}<\infty$; (iv) $\lim b_{n}=0$. Let $\left\{x_{n}\right\}$ be generated iteratively from arbitrary $x_{0} \in E$ by

$$
x_{n+1}=a_{n} x_{n}+b_{n} \eta_{n}+c_{n} u_{n}, \exists \eta_{n} \in T x_{n}, n \geq 0,
$$

where $\left\{u_{n}\right\}$ is an arbitrary bounded sequence in $E$. Then, there exists $\gamma_{0} \in \Re$ such that if $b_{n}+c_{n} \leq \gamma_{0} \forall n \geq 0$, then the sequence $\left\{x_{n}\right\}$ converges strongly to the unique fixed point of $T$.

Remark 3.9. In view of Remarks 3.5 and 3.6, our theorems significantly generalize the results of $\mathrm{Xu}$ et al., 15. Furthermore, since every $\phi$-hemi-contractive mapping 
is a generalized $\Phi$ hemi-contractive map, and every operator with bounded range is a generalized Lipschitz map, our theorems are significant improvements of the results of [8], which, as we had remarked, are themselves generalizations of important recent results.

\section{ACKNOWLEDGMENT}

The authors thank the referee for very useful comments.

\section{REFERENCES}

[1] F.E. Browder, Nonlinear operators and nonlinear equations of evolution in Banach spaces, Proc. of Symposia in Pure Math. Vol. XVIII, part 2 (1976). MR0405188 (53:8982)

[2] S.S. Chang, Y.J. Cho and H. Zhou, Iterative methods for nonlinear operator equations in Banach spaces, Nova Science Publishers, Inc., Huntington, NY (2002). MR2016857 (2005a:47130)

[3] S.S. Chang, Y.J. Cho and B.S. Lee, J.S. Jung, and S.M. Kang, Iterative approximations of fixed points and solutions for strongly accretive and strongly pseudocontractive mappings in Banach spaces, J. Math. Anal. Appl. 224(1998) 149-165. MR1632970 (99g:47146)

[4] C.E. Chidume, Iterative approximation of fixed points of Lipschitz pseudocontractive maps, Proc. Amer. Math. Soc. 129(2001), No. 8, 2245-2251. MR1823906 (2002e:47071)

[5] C.E. Chidume and H.Zegeye, Approximate fixed point sequences and convergence theorems for Lipschitz pseudocontractive maps, Proc. Amer. Math. Soc. 132(2004) 831-840. MR2019962 (2004j:47114)

[6] K. Deimling, Nonlinear functional analysis, Springer-Verlag, Berlin Heiderberg New York Tokyo (1980). MR0787404(86j:47001)

[7] F.Gu, Convergence theorems for $\Phi$-pseudo contractive type mappings in normed lnear spaces, Northeast Math. J. 17(3)(2001) 340-346. MR2011842 (2004h:47080)

[8] H. Hirano and Z. Huang, Convergence theorems for multi-valued $\Phi$-hemicontractive operators and $\Phi$-strongly accretive operators, Computers and Mathematics with Applications 46(2003) 1461-1471. MR2024221 (2004i:47136)

[9] S. Ishikawa, Fixed point by a new iteration method, Proc. Amer. Math. Soc. 44(1)(1974) 147-150. MR0336469 (49:1243)

[10] L.S. Liu, Ishikawa and Mann iterative process with errors for nonlinear strongly accretive mappings in Banach spaces, J. Math. Anal. Appl. 194(1995) 114-125. MR1353071(97g:47069)

[11] W.R. Mann, Mean value methods in iteration, Proc. Amer. Math. Soc. 4(1953) 506-510. MR0054846 (14:988f)

[12] B.E. Rhoades, Comments on two fixed point iteration methods, J. Math. Anal. Appl. 56(3)(1976) 741-750. MR0430880 (55:3885)

[13] Y. Xu, Ishikawa and Mann iterative processes with errors for nonlinear strongly accretive operator equations, J. Math. Anal. Appl. 224(1998) 91-101. MR1632966 (99g:47144)

[14] H.Y. Zhou and D.Q. Cheng, Iterative approximations of fixed points for nonlinear mappings of $\phi$-hemicontractive type in normed linear spaces, Math. Appl. 11(3)(1998) 118-121. MR:1675724 (99m:47061)

[15] Z.Q. Xue, H.Y. Zhou and Y.J. Cho, Iterative solutions of nonlinear equations for $m$ accretive operators in Banach spaces, J. Nonlinear and Convex Analysis 1(3) (2003) 313-320. MR.1807044 (2001j:47082)

The Abdus Salam International Centre for Theoretical Physics, Trieste, Italy

E-mail address: chidume@ictp.trieste.it

Department of Mathematics and Statistics, Auburn University, Auburn, Alabama

E-mail address: chidumeg@hotmail.com 